\title{
New generation anaplastic lymphoma kinase inhibitors
}

\author{
Angelo Delmonte, Marco Angelo Burgio, Alberto Verlicchi, Giuseppe Bronte, Paola Cravero, Paola Ulivi, \\ Giovanni Martinelli, Lucio Crinò
}

Thoracic Oncology Unit, Istituto Scientifico Romagnolo per lo Studio e la Cura dei Tumori (IRST), IRCCS, Meldola, Italy

Contributions: (I) Conception and design: A Delmonte, L Crinò; (II) Administrative support: MA Burgio, A Verlicchi, G Bronte, P Cravero, P Ulivi, G Martinelli; (III) Provision of study materials or patients: MA Burgio, A Verlicchi, G Bronte, P Cravero, P Ulivi, G Martinelli; (IV) Collection and assembly of data: A Delmonte, L Crinò; (V) Data analysis and interpretation: A Delmonte, L Crinò; (VI) Manuscript writing: All authors; (VII) Final approval of manuscript: All authors.

Correspondence to: Angelo Delmonte. Thoracic Oncology Unit, Istituto Scientifico Romagnolo per lo Studio e la Cura dei Tumori (IRST), IRCCS, via Maroncelli, 40, Meldola 47014, Italy. Email: angelo.delmonte@irst.emr.it.

\begin{abstract}
Anaplastic lymphoma kinase (ALK) gene translocations are pro-tumoral driver alterations that encompass 3-7\% of non-squamous non-small cell lung cancer (NSCLC) with specific, clinic and histologic features. The therapeutic strategy depends on anti-ALK tyrosine kinase inhibitors (TKIs) of which crizotinib was the first approved for clinical use. Despite its use improved significantly progression-free survival, overall response rate and duration of response of this illness, after a median period of 10.9 months all patients progress due to the development of acquired resistance mutations in the ALK tyrosine kinase domain in approximately one third of patients. Moreover, $60-90 \%$ of patients treated with crizotinib has a progression in the central nervous system (CNS) in absence of extracranial worsening of the disease. This is primarily attributed to poor CNS penetration by crizotinib as many pre-clinical and clinical models suggest. For instance, in order to overtake acquired resistance to crizotinib, prolong the control of the disease and manage CNS localizations, several II and III generation TKIs have been developed. Some of them were approved after the failure of crizotinib (ceritinib, alectinib, brigatinib and lorlatinib) and in first line setting (ceritinib, alectinib and brigatinib) while others are still under evaluation for TKI-naive patients such as lorlatinib, ensartinib and entrectinib. In this review we will discuss the most recent results of new TKIs in order to describe a fast growing therapeutic landscape in this setting.
\end{abstract}

Keywords: Anti-anaplastic lymphoma kinase tyrosine kinase inhibitors (anti-ALK TKIs); resistance; alectinib; ceritinib; brigatinib

Submitted Aug 22, 2019. Accepted for publication Sep 18, 2019.

doi: $10.21037 /$ tlcr.2019.09.14

View this article at: http://dx.doi.org/10.21037/tlcr.2019.09.14

Anaplastic lymphoma kinase (ALK) gene translocations are pro-tumoral driver alterations, present in $3-7 \%$ of nonsquamous non-small cell lung cancers (NSCLC) (1). Patients affected by this tumor are frequently young, women, with a limited or absent history of smoking and often with a histologic diagnosis of a signet-ring cell adenocarcinoma (2). In approximately $30 \%$ of these patients central nervous system (CNS) involvement is present at diagnosis (3). In this setting, the main therapeutic strategy is represented by anti-ALK rearrangement tyrosine kinase inhibitors (TKIs). Crizotinib, a I generation multi-TKI, initially developed as a mesenchymal-epithelial transition factor (MET) inhibitor, was the first used in clinic due to results of PROFILE trials showing median progression-free survival (mPFS), objective response rate (ORR) and median duration of response (mDOR) higher than those of chemotherapy in all lines of treatment $(4,5)$. Despite these results, after a median period of 10.9 months all patients progress due to the development, in approximately one third of patients, of acquired resistance mutations in the ALK tyrosine kinase domain, such as L1196M, G1269A, C1156Y, L1152R, G1202R, S1206Y, 1151Tins, F1174C, and D1203N. These 
Table 1 Targeting features of II and III generation anti-ALK TKIs

\begin{tabular}{lllll}
\hline Drug & Main target & IC $_{50}$ on ALK $(\mathrm{nM})$ & Activity against secondary ALK mutations & Ref. \\
\hline Alectinib & ALK, RET & 1.9 & $\begin{array}{l}\text { L1196M, G1269A, C1156Y, F1174L, 1151Tins, } \\
\text { L1152R }\end{array}$ & $(8,21)$ \\
Ceritinib & ALK, ROS1, IGF-1R & & L1196M, G1269A, S1206Y, I1171T \\
Brigatinib & ALK, EGFR L858R, ROS1, FLT3 & $1.5-12$ & All, including G1202R and L1196M \\
Lorlatinib & ALK, ROS1 & 1.3 & All, including G1202R & $(22)$ \\
Ensartinib & ALK, TPM3-TRKA, TRKC, ROS1, & $<4$ & F1174, C1156Y, L1196M, S1206R, T1151, \\
& EphA2, EphA1, EphB1, C-MET & & G1202R & $(25-27)$ \\
Entrectinib & ALK, ROS1, TRK & 12 & G1269A, C1156Y, L1196M & $(30)$ \\
\hline
\end{tabular}

ALK, Anaplastic lymphoma kinase; RET, rearranged during transfection; IGF-1R, insulin growth factor-1 receptor; EGFR, epidermal growth factor receptor; TKIs, tyrosine kinase inhibitors; $I_{50}$, half maximal inhibitory concentration; Ref., references.

alterations increase the affinity for adenosine triphosphate, inducing conformational changes with steric hindrance and interference with the downstream signaling pathway (6-11). Other mechanisms of resistance are amplification of ALK fusion gene, alone or in combination with other molecular alterations (6), or bypass pathways such as amplification of epidermal growth factor receptor (EGFR) or of insulin-like growth factor (IGF-1R) or cKIT mutation (12-14).

Among $60-90 \%$ of patients receiving crizotinib has a CNS progression which is the first site of relapse in $46 \%$ of cases without evidence of extracranial worsening $(3,15-17)$. This is attributed to poor CNS penetration by crizotinib as it is shown by the analysis of cerebro-spinal fluid from patients progressing in the CNS (18) and reinforced by the fact that ORR (33\% vs. 18\%) and prolongation in the median time to intracranial progression (13 vs. 7 months) are superior when crizotinib is given after radiotherapy than when is given alone (19) suggesting that prior radiation may probably increase the blood-brain barrier (BBB) permeability improving the CNS activity of crizotinib (20).

In order to overtake acquired resistance to crizotinib, prolong the control of the disease and manage CNS localizations, several II and III generation TKIs have been developed (Table 1), entering in clinic after the failure of crizotinib (ceritinib, alectinib, brigatinib and lorlatinib) (Table 2) and in first line setting (ceritinib, alectinib and brigatinib) (Table 3) while others are still under clinical evaluation for TKI-naive patients such as lorlatinib, ensartinib and entrectinib.

In this review we will discuss the most recent results of TKIs in order to describe a fast growing therapeutic landscape in this setting.

\section{Alectinib (R05424802/CH5424802, Alecensa)}

Alectinib is a strong and selective, competitive with ATP, II generation TKI mainly directed against ALK and Rearranged during Transfection (RET) gene rearrangements (48). In vitro it shows a threefold increase of ALK inhibition (53 $\mathrm{nM}$ alectinib versus $150.8 \mathrm{nM}$ crizotinib) by inducing caspase-mediated apoptosis in EML4-ALK cell lines, with a dose-dependent tumor growth inhibition (21). Its half maximal inhibitory concentration (IC50) is $1.9 \mathrm{nM}$ while that of crizotinib is $3 \mathrm{nM}$ (21). It is metabolized via hepatic CYP3A4 enzymes to M4, an active metabolite, and mainly excreted via the feces; mean terminal half-life is about 30 hours (49). Alectinib showed high efficacy against several crizotinib-resistant mutations in ALK, including L1196M, G1269A, C1156Y, F1174L, 1151Tins, and L1152R but not G1202R $(8,21)$. The drug strongly penetrate the CNS, as shown in intracranial tumor implantation model where it is able to inhibit the growth of ALK-positive CNS lesions; in this model p-glycoprotein (P-gp) doesn't transport the drug, showing a relevant CNSto-plasma ratio $(50)$.

Alectinib was initially evaluated in 2 phase I/II trial: in the first trial among 46 Japanese patients with ALK rearranged, untreated NSCLCs, 2 (4.3\%) complete responses (CR) and $41(89.1 \%)$ partial responses (PR) were seen with 7 having disease control for more than 6.5 months (51). The second phase I trial was conducted in US patients in crizotinib-resistant NSCLCs: objective responses $(\mathrm{OR})$ were noted in $55 \%$ of patients with $2 \% \mathrm{CR}$, $32 \%$ PR, $20 \%$ unconfirmed PR; $36 \%$ of patients had stable disease (SD). Eleven (52\%) out of 21 patients with baseline CNS metastases showed OR of which 6 (29\%) CR, 5 (24\%) 
Table 2 II and III generation TKIs in crizotinib pre-treated patients

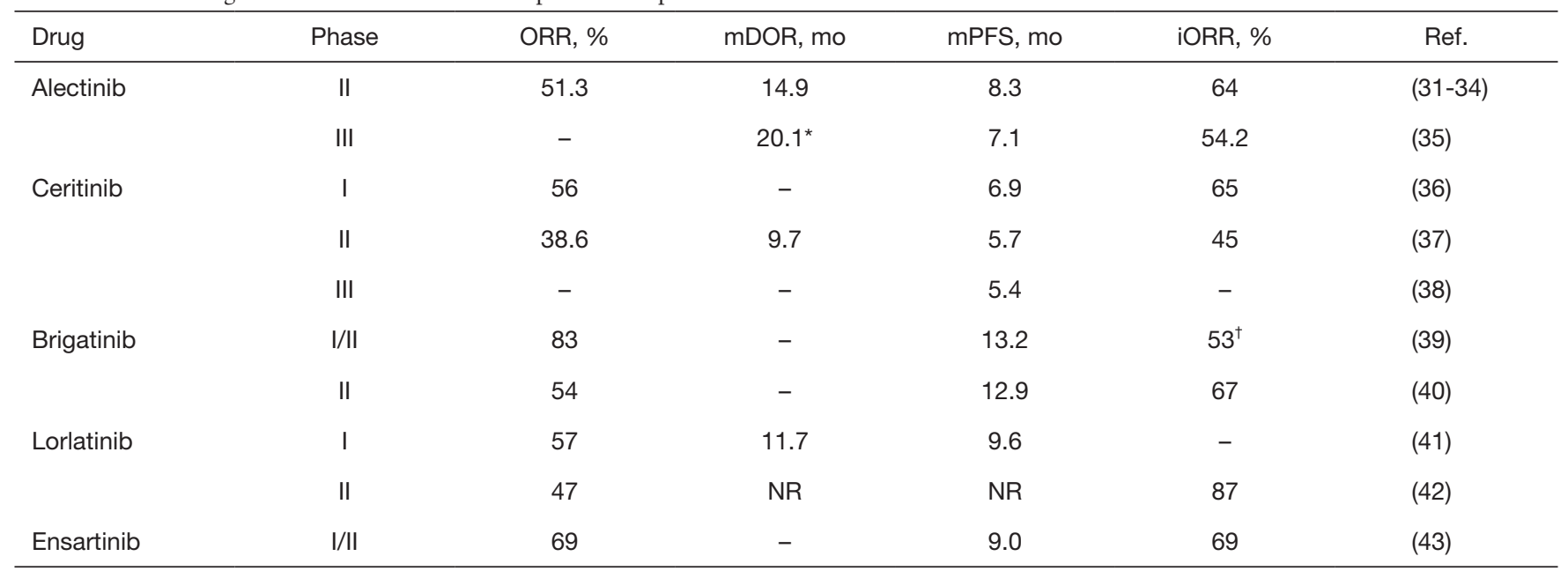

*, weeks; ${ }^{\dagger}$, in patients with measurable intracranial lesions. TKI, tyrosine kinase inhibitors; ORR, objective response rate; mDOR, median duration of response; mPFS, median progression-free survival; iORR, intracranial objective response rate; mo, months; Ref., reference; NR, not reached.

Table 3 Clinical trials in TKIs-naive patients

\begin{tabular}{|c|c|c|c|c|c|c|c|c|}
\hline Drug & Control arm & \multicolumn{2}{|c|}{ ORR, \% } & \multicolumn{2}{|c|}{ mPFS, mo } & \multicolumn{2}{|c|}{ iORR, \% } & Ref \\
\hline \multirow[t]{2}{*}{ Alectinib } & Crizotinib & 92 & 79 & NR & 10.2 & - & - & (44) \\
\hline & Crizotinib & 82.9 & 75.5 & NR & 11.1 & $85.7^{*}$ & $71.4^{\star}$ & (45) \\
\hline Ceritinib & Chemotherapy & 72.5 & 26.7 & 16.6 & 8.1 & 72.7 & 27.3 & (46) \\
\hline Lorlatinib & - & 90 & - & NR & - & 66.7 & - & (42) \\
\hline
\end{tabular}

${ }^{*}$, in patients with previous radiotherapy; ${ }^{\dagger}$, intended as estimated 12-month progression-free survival. TKls, tyrosine kinase inhibitors; ORR, objective response rate; Exp., experimental; mPFS, median progression-free survival; iORR, intracranial objective response rate; mo, months; Ref., reference; NR, not reached.

PR and 8 (38\%) SD (52). Twenty-six per cent of patients in the Japanese trial showed treatment-related adverse events (TRAEs) grade 3-4 toxicity and $8 \%$ in the American trial, being represented by decreased neutrophil count, increased blood creatine phosphokinase, $\gamma$-glutamyl transpeptidase and hypophosphataemia $(51,52)$.

In the crizotinib-resistant NSCLC setting, the pooled analysis of 2 phase II trials confirmed ORR of $51.3 \%$, disease control rate (DCR) of $78.8 \%$, mDOR of 14.9 months, mPFS of 8.3 months and median overall survival (mOS) of 26.0 months (31-33). The pooled analysis confirmed also the activity against CNS metastases with an intracranial ORR (iORR) of $64.0 \%$, DCR $90.0 \%$ and mDOR 10.8 months $\mathrm{iORR}$ was $35.8 \%$ in patients with prior radiotherapy and $58.5 \%$ in those untreated $(31,32,34)$. Most adverse events (AEs) were grade 1 to 2 (32); in $40 \%$ of patients grade 3 or higher AEs occurred, leading to treatment withdrawn or interruptions or modification in $6-33 \%$ of patients (33). The most common grade $3-4$ AEs were represented by changes in laboratory values, such as increase of blood creatine phosphokinase and of transaminases (31).

In the ALUR trial superiority of alectinib versus platinum-based chemotherapy in advanced, crizotinibresistant NSCLC was assessed by an independent review committee (IRC) in terms of PFS (7.1 vs. 1.6 months), ORR of CNS baseline lesions (54.2\% vs. no responses). The treatment period was longer in the alectinib arm (20.1 
versus 6.0 weeks). Alectinib showed $27.1 \%$ of grade $3 \mathrm{AEs}$ while chemotherapy $41.2 \%$; treatment discontinuation due to AEs was $5.7 \%$ with alectinib and $8.8 \%$ with chemotherapy (35).

Finally alectinib was evaluated in first line in untreated patients compared crizotinib in two phase III, randomized trials. The first one, the J-ALEX study, showed in a Japanese population a PFS not reached at the moment of publication in the alectinib arm while with crizotinib PFS was 10.2 months; OR was $92 \%$ versus $79 \%$ respectively (44). The second trial, the ALEX trial performed in the Caucasian population, showed similar activity with a 12 -month event-free survival rate of $68.4 \%$ with alectinib versus $48.7 \%$ with crizotinib. The hazard ratio (HR) for disease progression or death in the alectinib arm was 0.47 and the mPFS in the experimental arm not reached; ORR was $82.9 \%$ with alectinib and $75.5 \%$ $(\mathrm{P}=0.09)$ with crizotinib (45). Investigator-assessed PFS with alectinib was similar between patients with (HR 0.40, 95\% CI: 0.25-0.64) and without (HR 0.51, 95\% CI: $0.33-0.80, \mathrm{P}$ interaction $=0.36)$ CNS metastases at baseline, independently to a previous radiotherapy. Time to progression of brain metastases was longer in the alectinib arm and similar patients with and without baseline CNS metastases $(\mathrm{P}<0.0001)$; alectinib obtained an iORR of $85.7 \%$ in patients who underwent to radiotherapy and of $78.6 \%$ in not treated CNS lesions while crizotinib an iORR of $71.4 \%$ and $40.0 \%$, respectively (53). In the two trials grade 3-5 AEs were $41 \%$ with alectinib and $50 \%$ with crizotinib (45).

\section{Ceritinib (LDK378, Zykadia)}

Ceritinib is a potent and selective, ATP-competitive, oral TKI, directed against rearrangement of ALK and protooncogene receptor tyrosine kinase (ROS1) and alterations of insulin growth factor-1 receptor (IGF-1R). It's able to inhibit ALK with 20-fold greater potency than crizotinib in enzymatic assays with an IC50 of $0.15 \mathrm{nM}$ with a more durable antitumor activity (22). Cytochrome CYP3A metabolizes ceritinib which is mainly excreted via feces $(92.3 \%$ vs. $1.3 \%$ via urine) (54). Ceritinib is highly active against the most common crizotinib-resistance mutations, including L1196M, G1269A, S1206Y and I1171T (22). Finally a tissue distribution study in rats shows that ceritinib cross the blood brain barrier, with a brain-toblood exposure ratio of approximately $15 \%$ (36).

The development of ceritinib was conducted in the ASCEND program, starting with the phase I trial conducted in TKI-naive and TKI-pretreated patients: ORR was $72.0 \%$ and $56.0 \%$, respectively; mPFS was 18.4 and 6.9 months. Among patients with confirmed brain intracranial involvement, DCR was $79 \%$ in TKI-naive and $65 \%$ TKI-pretreated patients. The most frequent grade 3-4 AEs were increased alanine aminotransferase (30\%), increased aspartate aminotransferase (10\%), diarrhoea and nausea in $6 \%$ of patients (36).

The phase II (ASCEND-2) trial involved patients progressing after crizotinib and 2 or more other treatment regimens, encompassing also people with asymptomatic or neurologically stable baseline CNS metastases: ORR was $38.6 \%$, DCR $77.1 \%$, median time to response 1.8 months, mDOR 9.7 months and mPFS 5.7 months. Among 100 patients with baseline brain metastases, iORR was $45.0 \%$. Adverse events were in the majority grade 1 or 2 ; the most frequent were nausea $(81.4 \%)$, diarrhea $(80.0 \%)$, and vomiting $(62.9 \%)$. Improvement in quality of life was shown by patient-reported outcomes (37).

In the phase III (ASCEND-4) trial treatment-naive population was randomized to receive ceritinib or platinumbased chemotherapy: blinded IRC showed a mPFS of 16.6 months with ceritinib vs. 8.1 months with chemotherapy (HR 0.55; 95\% CI: 0.42-0.73; $\mathrm{P}<0.00001$ ). Diarrhoea $(85 \%)$, nausea $(69 \%)$, vomiting $(66 \%)$ and increase in alanine aminotransferase $(60 \%)$ were the most frequent AEs with ceritinib (46). Confirmation of those results were obtained in the phase III trial (ASCEND-5) in which patients at failure of chemotherapy and crizotinib were randomized to receive ceritinib or chemotherapy with mPFS of 5.4 months (95\% CI: 4.1-6.9 months) in ceritinib arm and 1.6 months (1.4-2.8 months). Forty-three per cent of patients receiving ceritinib experienced serious adverse events (SAEs) vs. 32\% in the chemotherapy group. Increased alanine aminotransferase concentration (21\%), increased $\gamma$ glutamyltransferase concentration (21\%) and increased aspartate aminotransferase concentration (14\%) were the most represented grade 3-4 AEs in patients treated with ceritinib (38).

Given the high incidence of AEs, the phase Ib (ASCEND-8) trial compared in term of activity and tolerability 3 different doses of ceritinib after a light breakfast instead at fasted state showing that the smallest one, $450 \mathrm{mg}$ with food, demonstrated pharmacokinetic parameters comparable to those obtained with the registative dose, $750 \mathrm{mg}$ fasted (55). The arm receiving $450 \mathrm{mg}$ showed the lowest proportion of patients with dose reductions, of patients with gastrointestinal toxicities and 
the longest mDOR; ORR was similar in the 3 arms (56).

\section{Brigatinib (AP26113, Alunbrig)}

Brigatinib is a synthetic, oral, II generation TKI able to inhibit ALK kinase with a stronger potency than crizotinib (23), exerting dual inhibition of ALK and EGFR (epidermal growth factor receptor) (L858R) in humans (24). It shows high selectivity in inhibiting ROS1, FLT3, and mutant variants of FLT3 (D835Y) but low activity against EGFR with a T790M resistance mutation (L858R/T790M), native EGFR, IGF1R (23). Brigatinib is mainly metabolised by CYP2C 8 and CYP3A4 in vitro. About $3.5 \%$ of brigatinib gets metabolised to its primary metabolite, less potent, and $92 \%$ remain unchanged. Hepatic elimination is the major route of excretion, $65 \%$ of administered dose being recovered from feces and $25 \%$ from urine $(57,58)$. Steady state maximum concentration is reduced to be about $13 \%$ when administered after high fat meal (59). In orthotopic brain tumor model, brigatinib showed a prolongation of median survival of mice and a significant reduction of intracranial tumor burden more actively than crizotinib (23). Brigatinib displays an inhibitory profile against all 17 known resistance mutations of ALK, including the G1202R and L1196M, superior than that of crizotinib, ceritinib and alectinib (23).

Brigatinib was initially evaluated in a phase I/II trial. After a $3+3$ dose escalation phase in which the doselimiting toxicities (DLT) were increased transaminases and dyspnoea, the phase 2 part evaluated 3 oral once-daily regimens into 5 cohorts: TKI-naive NSCLC (cohort 1), crizotinib-pretreated NSCLC (cohort 2), EGFR T790Mpositive NSCLC resistant to previous anti-EGFR TKI (cohort 3), solid tumors with abnormalities of the molecular pathways, targets of the drug (cohort 4) and crizotinibnaive or crizotinib-treated NSCLC with active, measurable, intracranial CNS metastases (cohort 5). Responses were seen only in NSCLCs: ORR was 100\% in cohort 1, 74\% in cohort 2 and $83 \%$ in cohort 5 . The mPFS was not reached in crizotinib-naive patients and 13.2 months in crizotinibpretreated patients; probability of OS at 1 year was $100 \%$ and $78 \%$, respectively. In the cohort 5 iORR was $53 \%$ in patients with CNS measurable lesions and $35 \%$ in the nonmeasurable disease group. Intracranial mPFS was 15.6 months, increasing to 22.3 months for patients with no prior radiotherapy. The most active dose was represented by $180 \mathrm{mg}$ daily but the occurrence of pulmonary events within the first week of treatment, higher with this dose and causing dose interruption, leaded to a schedule with a 7-day lead-in dose of $90 \mathrm{mg}$ daily followed by $180 \mathrm{mg}$ daily continuously. Across all doses increased lipase concentration (9\%), dyspnoea (6\%) and hypertension (5\%) were the most represented grade 3-4 TRAEs (39).

Those data were subsequently confirmed in the phase II, ALTA trial in which patients progressing to crizotinib were randomized to receive brigatinib at $90 \mathrm{mg}$ daily in arm A or $180 \mathrm{mg}$ daily with a 7-day lead-in at $90 \mathrm{mg}$ in arm B: ORR was $45 \%$ in arm A and $54 \%$ in arm B; iORR in patients with baseline CNS target lesions $42 \%$ (11 of 26 patients) and 67\% (12 of 18 patients) respectively; PFS 9.2 months and 12.9 months respectively. Common TRAEs were mainly grades 1-2 and were mainly represented by nausea, diarrhea, headache and cough (40). Given these results arm $B$ schedule of brigatinib was compared to crizotinib in the first line setting in the phase 3 ALTA$1 \mathrm{~L}$ trial. At the first interim analysis the rate of PFS was higher in the experimental arm with an estimated 12-month PFS of $67 \%$ compared to $43 \%$ (HR 0.49 ; $\mathrm{P}<0.001$ ) in the crizotinib arm; ORR was $71 \%$ with brigatinib and $60 \%$ with crizotinib; iORR among patients with measurable lesions was $78 \%$ and $29 \%$, respectively, without new safety data (47).

\section{Lorlatinib (PF-06463922, Lorviqua)}

Lorlatinib is a III generation macrocyclic, ATP-competitive, oral TKI showing potent inhibition against ALK (Ki 1/4 $0.70 \mathrm{nM}$ ) and ROS1 rearrangement (Ki 1/4 $0.025 \mathrm{nM}$ ) $(25,26)$. In vitro and in vivo metabolite assays, lorlatinib was able to alter PK of concomitant drugs substrate of the CYP/CYP450 pathways, leading to the contraindication to CYP3A inhibitors since 12 days prior to the first dose of lorlatinib in clinical trials. It is active against all known resistant mutants for I and II generation ALK inhibitors including G1202R (27): in fact mean IC50 values against G1202R of lorlatinib, crizotinib, ceritinib and alectinib were $80,560,309$, and $595 \mathrm{nM}$, respectively, permitting to conclude that only lorlatinib inhibits this target (27). Lorlatinib was developed from crizotinib in order to facilitate the CNS penetration and to reduce the P-gpdependent efflux $(25,60)$. In fact BBB penetration was shown by assays in a non-human primate model while in mouse models the systemic and intracranial efficacy of lorlatinib increased the survival (61).

The drug was evaluated initially in a phase I trial encompassing patients with advanced, NSCLC of which ALK-rearranged patients, TKI-naive or progressing 
after at least 1 previous TKI, represented the $76 \%$ of trial population. In this group ORR was $46 \%$ with $7 \%$ CR, 39\% PR and 20\% SD; ORR 57\% in patients with a previous ALK TKI and $42 \%$ in those treated with 2 or more ALK TKIs. The estimated mDOR was 11.7 months for those previously treated; mPFS was 9.6 months being 13.5 months for patients treated with 1 previous TKI and 9.2 in those with 2 or more TKIs. The most common TRAEs were hypercholesterolaemia (72\%), hypertriglyceridaemia (39\%), peripheral neuropathy (39\%) and peripheral oedema (39\%) but the only DLT was grade 2 neurocognitive alterations (slowed speech, mentation, and word-finding difficulty) (41).

These data are at present under evaluation in a phase II trial involving a similar population in 6 different expansion cohorts (EXP1-6) according to previous treatments and the status of molecular drivers. Preliminary data were published concerning ALK-rearranged NSCLC cohorts encompassing patients TKI naïve (EXP1), crizotinibpretreated without (EXP2) or with (EXP3A) previous chemotherapy, with 1 previous non-crizotinib TKI with or without chemotherapy (EXP3B) and with 2 (EXP4) or 3 (EXP5) previous TKIs with or without chemotherapy. In EXP1 OR was $90.0 \%$; in EXP2-5 OR was $47.0 \%$ being $69.5 \%$ in EXP2-3A, $32.1 \%$ in EXP $3 \mathrm{~B}$ and $38.7 \%$ in EXP4-5. In patients with CNS measurable lesions iORR was $66.7 \%$ in EXP1, 87.0\% in EXP2-3A, 55.6\% in EXP3B and $53.1 \%$ in EXP4-5. In all the expansions mDOR was not reached nor in the intracranial neither in the extracranial disease; mPFS was not reached in EXP 1 and in EXP2-3A but it was 5.5 months (in EXP3B and 6.9 months in EXP4-5. The most common TRAEs across all patients were hypercholesterolaemia ( $81 \%$ overall and $16 \%$ grade $3-4)$ and hypertriglyceridaemia (60\% overall and $16 \%$ grade 3-4). Serious TRAEs occurred in $7 \%$ of patients and only $3 \%$ permanently discontinued treatment because of iatrogenic toxicity. CNS effects of any cause were reported in $39 \%$ of patients, including changes in cognitive function $(23 \%), \operatorname{mood}(22 \%)$ and speech (8\%) and were grade 1 or 2 in severity, transient, intermittent, and reversible after dose modifications (42).

Finally the drug has been compared to crizotinib in the Phase III CROWN study (NCT03052608) of which the enrollment was recently concluded.

\section{Ensartinib (X-396)}

Ensartinib (X-396) is a novel II generation, aminopyridazine- based ALK-TKI able to inhibit both wild-type ALK and all evaluated ALK variants (F1174, C1156Y, L1196M, S1206R, T1151, and G1202R mutants) with in vitro IC50 of $<4 \mathrm{nM}$; it also potently inhibits TPM3-TRKA, TRKC, ROS1, EphA2, EphA1, EphB1 and c-MET (28). Preclinical data demonstrated increased potency of the drug as compared with crizotinib and other II generation TKIs (29).

Safety and efficacy were evaluated in a phase I/II trial: in the phase I part the maximum tolerated dose was not reached and the most common TRAEs were rash (56\%), nausea (36\%), pruritus (28\%), vomiting (26\%), and fatigue (22\%). Differently to other II generation ALK TKIs, ensartinib induced grade $3-4$ toxicity in $23 \%$ of patients mainly represented by rash and pruritus. In fact the concentration of ensartinib was 9.0 times higher in the skin than in the plasma at 12 hours after its single dose. In the phase II part 60 patients with advanced ALK-rearranged NSCLC were evaluated: the DCR was $81.7 \%$, ORR $60 \%$ and mPFS 9.2 months. In TKI-naive patients ORR was $80 \%$ and mPFS 26.2 months; in those progressing to crizotinib ORR was $69 \%$ and mPFS 9.0 months; in patients progressing after 2 lines of TKIs mPFS was 1.9 months (62). Twenty-six patients had at baseline brain metastasis with iORR of $69 \%$, including $1 \mathrm{CR}, 31 \% \mathrm{SD}$; DCR was $100 \%$ in patients with CNS target lesions. In those with unmeasurable lesions $1 \mathrm{CR}$ was achieved and 8 pts had SD (43).

Data from the phase III trial comparing ensartinib to crizotinib, which have closed the enrollment in 2018, are awaited.

\section{Entrectinib (RXDX-101)}

Entrectinib is a potent, orally available, ATP competitive inhibitor of the ALK, ROS1 and TRK family rearrangements. In biochemical assay, entrectinib inhibits ALK and ROS1 with IC50 values of 12 and $7 \mathrm{nmol} / \mathrm{L}$, respectively. In in vitro and in vivo models entrectinib showed activity against ALK-rearranged NSCLC, efficiently crossed the BBB with drug levels within the brain comparable with or exceeding plasma levels and showed a strong intracranial activity. It showed good antiproliferative activity with strong activity against G1269A mutation, slight loss of potency in presence of C1156Y and L1196M ALK-resistance mutations and minimal activity on G1202R mutant (30).

Different schedules of the drug were evaluated into 2 phase I/II trials, the Alka-372-001 trial and the 
STARTRK-1, enrolling solid tumors with rearrangements of TRK family, ROS1 or ALK. In the 2 trials 27 patients with ALK-rearranged solid tumors were present. Among the 19 patients, pretreated with 1 or more ALK TKIs, no response to entrectinib was seen. In the remaining 7 TKInaive patients ORR was 57\% (95\% CI: $25-84 \%$ ) and responses were observed in ALK -rearranged NSCLC, renal cell carcinoma and colorectal cancer. Median DOR in ALK population was 7.4 months (95\% CI: 3.7 monthsnot reached) with a mPFS of 8.3 months $(95 \%$ CI: 4.6-12 months); mOS wasn't reached (95\% CI: 19 monthsnot reached) and the proportion of patients surviving at 12 months was $89.4 \%$ (95\% CI: $75.5-100 \%$ ). No DLTs or significant safety issues were reported in the 2 trials with the majority AEs of only grade 1 or 2 (63-65).

\section{Conclusions}

ALK rearranged NSCLC is a relatively rare disease for which a rich amount of new targeted drugs is present with an activity, both in terms of extra and intracranial disease, stronger than that of the first in class, first generation TKI crizotinib. These drugs differ for the targeting profile, especially against resistance mutations to previous TKIs, suggesting that their use may be defined according to the biological evolution of the tumor. For this reason clarification of several molecular aspects is strongly required. Moreover they differ for the safety profile showing specific toxicity features that may influence their choice according to patient characteristics. For instance, the knowledge of these biological and clinical aspects is necessary to define the best strategy of therapeutic sequence in order to give to patients the strongest and longest response, with the best quality of life.

\section{Acknowledgments}

None.

\section{Footnote}

Conflicts of Interest: The authors have no conflicts of interest to declare.

Ethical Statement: The authors are accountable for all aspects of the work in ensuring that questions related to the accuracy or integrity of any part of the work are appropriately investigated and resolved.

\section{References}

1. Soda M, Choi YL, Enomoto M, et al. Identification of the transforming EML4-ALK fusion gene in non-small-cell lung cancer. Nature 2007;448:561-6.

2. Kim H, Chung JH. Overview of clinicopathologic features of ALK-rearranged lung adenocarcinoma and current diagnostic testing for ALK rearrangement. Transl Lung Cancer Res 2015;4:149-5.

3. Johung KL, Yeh N, Desai NB, et al. Extended Survival and Prognostic Factors for Patients With ALK-Rearranged Non-Small-Cell Lung Cancer and Brain Metastasis. J Clin Oncol 2016;34:123-9.

4. Solomon BJ, Mok T, Kim DW, et al. First-line crizotinib versus chemotherapy in ALK-positive lung cancer. $\mathrm{N}$ Engl J Med 2014;371:2167-77.

5. Shaw AT, Kim DW, Nakagawa K, et al. Crizotinib versus chemotherapy in advanced ALK-positive lung cancer. $\mathrm{N}$ Engl J Med 2013;368:2385-94.

6. Doebele RC, Pilling AB, Aisner DL, et al. Mechanisms of resistance to crizotinib in patients with ALK gene rearranged non-small cell lung cancer. Clin Cancer Res 2012;18:1472-82.

7. Choi YL, Soda M, Yamashita Y, et al. EML4- ALK mutations in lung cancer that confer resistance to ALK inhibitors. N Engl J Med 2010;363:1734-9.

8. Kodama T, Tsukaguchi T, Yoshida M, et al. Selective ALK inhibitor alectinib with potent antitumor activity in models of crizotinib resistance. Cancer Lett 2014;351:215-21.

9. Rolfo C, Passiglia F, Castiglia M, et al. ALK and crizotinib: after the honeymoon... what else? Resistance mechanisms and new therapies to overcome it. Transl Lung Cancer Res 2014;3:250-61.

10. Katayama R, Shaw AT, Khan TM, et al. Mechanisms of acquired crizotinib resistance in ALK- rearranged lung cancers. Sci Transl Med 2012;4:120ra17.

11. Sasaki T, Koivunen J, Ogino A, et al. A novel ALK secondary mutation and EGFR signaling cause resistance to ALK kinase inhibitors. Cancer Res 2011;71:6051-60.

12. Katayama R, Khan TM, Benes C, et al. Therapeutic strategies to overcome crizotinib resistance in non-small cell lung cancers harboring the fusion oncogene EML4ALK. Proc Natl Acad Sci U S A 2011;108:7535-40.

13. Crystal AS, Shaw AT, Sequist LV, et al. Patient-derived models of acquired resistance can identify effective drug combinations for cancer. Science 2014;346:1480-6.

14. Lovly CM, McDonald NT, Chen H, et al. Rationale for co-targeting IGF-1R and ALK in ALK fusion-positive 
lung cancer. Nat Med 2014;20:1027-34.

15. Gainor JF, Sherman CA, Willoughby K, et al. Alectinib salvages CNS relapses in ALK-positive lung cancer patients previously treated with crizotinib and ceritinib. J Thorac Oncol 2015;10:232-6.

16. Weickhardt AJ, Scheier B, Burke JM, et al. Local ablative therapy of oligoprogressive disease prolongs disease control by tyrosine kinase inhibitors in oncogeneaddicted non-small-cell lung cancer. J Thorac Oncol 2012;7:1807-14.

17. Ou SH, Jänne PA, Bartlett $\mathrm{CH}$, et al. Clinical benefit of continuing ALK inhibition with crizotinib beyond initial disease progression in patients with advanced ALKpositive NSCLC. Ann Oncol 2014;25:415-22.

18. Costa DB, Kobayashi S, Pandya SS, et al. CSF concentration of the anaplastic lymphoma kinase inhibitor crizotinib. J Clin Oncol 2011;29:e443-5.

19. Costa DB, Shaw AT, Ou SH, et al. Clinical Experience With Crizotinib in Patients With Advanced ALKRearranged Non-Small-Cell Lung Cancer and Brain Metastases. J Clin Oncol 2015;33:1881-8.

20. Metro G, Lunardi G, Floridi P, et al. CSF Concentration of Crizotinib in Two ALK-Positive Non-Small-Cell Lung Cancer Patients with CNS Metastases Deriving Clinical Benefit from Treatment. J Thorac Oncol 2015;10:e26-7.

21. Sakamoto H, Tsukaguchi T, Hiroshima S, et al. CH5424802, a selective ALK inhibitor capable of blocking the resistant gatekeeper mutant. Cancer Cell 2011;19:679-90.

22. Friboulet L, Li N, Katayama R, et al. The ALK inhibitor ceritinib overcomes crizotinib resistance in non-small cell lung cancer. Cancer Discov 2014;4:662-73.

23. Zhang, S., Anjum, R, Squillace R, et al. The potent ALK inhibitor brigatinib (AP26113) overcomes mechanisms of resistance to first-and second generation ALK inhibitors in preclinical models. Clin Cancer Res 2016;22:5527-38.

24. Huang WS, Liu S, Zou D, et al. Discovery of brigatinib (AP26113), a phosphine oxidecontaining, potent, orally active inhibitor of anaplastic lymphoma kinase. J Med Chem 2016;59:4948-64.

25. Johnson TW, Richardson PF, Bailey S, et al. Discovery of (10R)-7-amino-12-fluoro-2,10,16-trimethyl-15-oxo-10,15, 16,17-tetrahydro-2H-8,4-(metheno)pyrazolo[4,3-h] $[2,5,11]$-benzoxadiazacyclotetradecine-3-carbonitrile (PF-06463922), a macrocyclic inhibitor of anaplastic lymphoma kinase (ALK) and c-ros oncogene 1 (ROS1) with preclinical brain exposure and broad-spectrum potency against ALK-resistant mutations, J Med Chem
2014;57:4720-44.

26. Basit S, Ashraf Z, Lee K, et al. First macrocyclic 3rdgeneration ALK inhibitor for treatment of ALK/ ROS1 cancer: clinical and designing strategy update of lorlatinnib. Eur J Med Chem 2017;134:348-56.

27. Zou HY, Friboulet L, Kodack DP, et al. PF-06463922, an ALK/ROS1 inhibitor, overcomes resistance to first and second generation ALK inhibitors in preclinical models. Cancer Cell 2015;28:70-81.

28. Li T, Ma W, Tian EC. Ensartinib (X-396): what does it add for patients with ALK-rearranged NSCLC. Chin Clin Oncol 2019;8:S4.

29. Singhi EK, Horn L. Background and rationale of the eXalt3 trial investigating X-396 in the treatment of ALK+ non-small-cell lung cancer. Future Oncol 2018;14:1781-7.

30. Ardini E, Menichincheri M, Banfi P, et al. Entrectinib, a Pan-TRK, ROS1, and ALK Inhibitor with Activity in Multiple Molecularly Defined Cancer Indications. Mol Cancer Ther 2016;15:628-39.

31. Shaw AT, Gandhi L, Gadgeel S, et al. Alectinib in ALKpositive, crizotinib-resistant, non-small-cell lung cancer: a single-group, multicentre, phase 2 trial. Lancet Oncol 2016;17:234-42.

32. Ou SH, Ahn JS, De Petris L, et al. Alectinib in Crizotinib-Refractory ALK-Rearranged Non-SmallCell Lung Cancer: A Phase II Global Study. J Clin Oncol 2016;34:661-8.

33. Yang JC, Ou SI, De Petris L, et al. Pooled Systemic Efficacy and Safety Data from the Pivotal Phase II Studies (NP28673 and NP28761) of Alectinib in ALKpositive Non-Small Cell Lung Cancer. J Thorac Oncol 2017;12:1552-60.

34. Gadgeel SM, Shaw A, Govindan R, et al. Pooled Analysis of CNS Response to Alectinib in Two Studies of Pretreated Patients With ALK-Positive Non-Small-Cell Lung Cancer. J Clin Oncol 2016;34:4079-85.

35. Novello S, Mazieres J, Oh IJ, et al. Alectinib versus chemotherapy in crizotinib pretreated anaplastic lymphoma kinase (ALK)-positive non-small-cell lung cancer: results from the phase III. Ann Oncol 2018;29:1409-16.

36. Kim DW, Mehr R, Tan DSW, et al. Activity and safety of ceritinib in patients with ALK-rearranged non-smallcell lung cancer (ASCEND-1): updated results from the multicentre, open-label, phase 1 trial. Lancet Oncol 2016;17:452-63.

37. Crinò L, Ahn M, De Marinis F, et al. Multicenter Phase II Study of Whole-Body and Intracranial Activity With 
Ceritinib in Patients With ALK-Rearranged Non-SmallCell Lung Cancer Previously Treated With Chemotherapy and Crizotinib: Results From ASCEND-2. J Clin Oncol 2016;34:2866-73.

38. Shaw AT, Kim TM, Crinò L, et al. Ceritinib versus chemotherapy in patients with ALK-rearranged nonsmall-cell lung cancer previously given chemotherapy and crizotinib (ASCEND-5): a randomised, controlled, openlabel, phase 3 trial. Lancet Oncol 2017;18:874-86.

39. Gettinger SN, Bazhenova LA, Langer CJ, et al. Activity and safety of brigatinib in ALK-rearranged non-small-cell lung cancer and other malignancies: a single-arm, openlabel, phase 1/2 trial. Lancet Oncol 2016;17:1683-96.

40. Kim DW, Tiseo M, Ahn MJ, et al. Brigatinib in Patients With Crizotinib-Refractory Anaplastic Lymphoma KinasePositive Non-Small-Cell Lung Cancer: A Randomized, Multicenter Phase II Trial. J Clin Oncol 2017;35:2490-98.

41. Shaw AT, Felip E, Bauer TM, et al. Lorlatinib in nonsmall-cell lung cancer with ALK or ROS1 rearrangement: an international, multicentre, open-label, single-arm firstin-man phase 1 trial. Lancet Oncol 2017;18:1590-99.

42. Solomon BJ, Besse B, Bauer TM, et al. Lorlatinib in patients with ALK-positive non-small-cell lung cancer: results from a global phase 2 study. Lancet Oncol 2018;19:1654-67.

43. Reckamp KL, Wakelee HA, Patel S, et al. CNS activity of ensartinib in ALK+ non-small cell lung cancer (NSCLC) patients (pts). Ann Oncol 2017;28:ii31.

44. Hida T, Nokihara H, Kondo M, et al. Alectinib versus crizotinib in patients with ALK-positive non-small-cell lung cancer (J-ALEX): an open-label, randomised phase 3 trial. Lancet 2017;390:29-39.

45. Peters S, Camidge R, Alice T. Shaw, et al. Alectinib versus Crizotinib in Untreated ALK-Positive Non-Small-Cell Lung Cancer. N Engl J Med 2017;377:829-38.

46. Soria JC, Tan D, Chiari R, et al. First-line ceritinib versus platinum-based chemotherapy in advanced ALKrearranged non-small-cell lung cancer (ASCEND-4): a randomised, open-label, phase 3 study. Lancet 2017;389:917-29.

47. Camidge DR, Kim HR, Ahn MJ, et al. Brigatinib versus Crizotinib in ALK-Positive Non-Small-Cell Lung Cancer. N Engl J Med 2018;379:2027-39.

48. Kodama T, Tsukaguchi T, Satoh Y, et al. Alectinib shows potent antitumor activity against RET-rearranged non small cell lung cancer. Mol Cancer Ther 2014;13:2910-18.

49. Genentech. (2016). Alecensa (alectinib) package insert. Available online: http://www.gene.com/download/pdf/ alecensa_prescribing.pdf

50. Kodama T, Hasegawa M, Takanashi K, et al. Antitumor activity of the selective ALK inhibitor alectinib in models of intracranial metastases. Cancer Chemother Pharmacol 2014;74:1023-8.

51. Seto T, Kiura K. CH5424802 (RO5424802) for patients with ALK-rearranged advanced non-small-cell lung cancer (AF-001JP study): a single-arm, open-label, phase 1-2 study. Lancet Oncol 2013;14:590-8.

52. Gadgeel SM, Gandhi L, Riely GJ, et al. Safety and activity of alectinib against systemic disease and brain metastases in patients with crizotinib-resistant ALK-rearranged non-small-cell lung cancer (AF-002JG): results from the dose-finding portion of a phase 1/2 study. Lancet Oncol 2014;15:1119-28.

53. Gadgeel S, Peters S, Mok T, et al. Alectinib versus crizotinib in treatment-naive anaplastic lymphoma kinase-positive (ALK) non-small-cell lung cancer: CNS efficacy results from the ALEX study. Ann Oncol 2018;29:2214-22.

54. Zykadia (ceritinib) prescribing information. East Hanover, NJ, USA: Novartis; 2015.

55. Cho BC, Kim DW, Bearz A, et al. ASCEND-8:A Randomized Phase 1 Study of Ceritinib, $450 \mathrm{mg}$ or 600 $\mathrm{mg}$, Taken with a Low-Fat Meal versus $750 \mathrm{mg}$ in Fasted State in Patients with Anaplastic Lymphoma Kinase (ALK)-Rearranged Metastatic Non-Small Cell Lung Cancer (NSCLC). J Thorac Oncol 2017;12:1357-67.

56. Cho BC, Obermannova R, Bearz A, et al. Efficacy and Safety of Ceritinib (450 mg/day or $600 \mathrm{mg} /$ day) With Food vs $750 \mathrm{mg}$ /day Fasted in Patients With ALK-Positive NSCLC: Primary Efficacy Results From ASCEND-8 Study. J Thorac Oncol 2019;14:1255-65.

57. Shakespeare W, Fantin V, Wang F, et al. Abstract 3738: Discovery of potent and selective orally active inhibitors of anaplastic lymphoma kinase (ALK). Cancer Res 2009;69:3738

58. ARIAD Pharmaceuticals Inc., 2017. ALUNBRIGTM (brigatinib): US prescribing information. Available online: https://www.accessdata.fda.gov/drugsatfda_docs/ label/2017/ 208772lbl.pdf (accessed 14.02.18).

59. Kadi AA, Attwa MW, Darwish HW, et al. LC-ESIMS/MS reveals the formation of reactive intermediates in brigatinib metabolism: elucidation of bioactivation pathways. RSC Adv 2018;8:1182-90.

60. Huang Q, Johnson TW, Bailey S, et al. Design of potent and selective inhibitors to overcome clinical anaplastic lymphoma kinase mutations resistant to crizotinib. J Med 
Chem 2014;57:1170-87.

61. Collier TL, Normandin MD, Stephenson NA, et al. Synthesis and preliminary PET imaging of $11 \mathrm{C}$ and $18 \mathrm{~F}$ isotopologues of the ROS1/ALK inhibitor lorlatinib. Nat Commun 2017;8:15761.

62. Horn L, Infante JR, Reckamp KL, et al. Ensartinib (X-396) in ALK-Positive Non-Small Cell Lung Cancer: Results from a First-in-Human Phase I/II, Multicenter Study. Clin Cancer Res 2018;24:2771-9.

63. De Braud F, Niger M, Damian S, et al. Alka-372-001: First-in-human, phase I study of entrectinib - an oral pantrk, ROS1, and ALK inhibitor - in patients with advanced

Cite this article as: Delmonte A, Burgio MA, Verlicchi A, Bronte G, Cravero P, Ulivi P, Martinelli G, Crinò L. New generation anaplastic lymphoma kinase inhibitors. Transl Lung Cancer Res 2019;8(Suppl 3):S280-S289. doi: 10.21037/ tlcr.2019.09.14 solid tumors with relevant molecular alterations. J Clin Oncol 2015;33:abstr 2517.

64. Patel MR, Bauer TM, Liu SV, et al. STARTRK-1: Phase 1/2a study of entrectinib, an oral Pan-Trk, ROS1, and ALK inhibitor, in patients with advanced solid tumors with relevant molecular alterations. J Clin Oncol 2015;33:abstr 2596.

65. Drilon A, Siena S, Ou S, et al. Safety and Antitumor Activity of the Multitargeted Pan-TRK, ROS1, and ALK Inhibitor Entrectinib: Combined Results from Two Phase I Trials (ALKA-372-001 and STARTRK-1). Cancer Discov 2017;7:400-9. 